\title{
DKK und INTERGEO in Stuttgart
}

\section{Mark Vetter $^{1}$}

Published online: 16 August 2019

(c) The Author(s) 2019

Liebe Leserinnen und Leser,

vom 17.-19.9.2019 findet in Stuttgart der DKK mit der INTERGEO statt. Jochen Schiewe, unser Vizepräsident national, konnte aufgrund der zahlreichen Vortragseinreichungen ein vielseitiges und spannendes Programm zusammenstellen. Das Hauptthema des Kongresses wird mit der Metapher „mit offenen Karten spielen“ treffend beschrieben. Offene Geodaten, häufig auch „freiwillig“ entstanden (VGI, ,,volunteered geographic information“), spielen in der Anwendung immer häufiger eine Rolle. Das Wikipedia-Projekt für Karten, OpenStreetMap, gibt es bereits seit gut 15 Jahren. Kommunen, NGOs, Forschungsund Bildungseinrichtungen verwenden diese offenen Karten für verschiedene Zwecke - und dies mit unterschiedlichsten Zielen. Die Betreiber der OpenStreetMap-Plattform, die OpenStreetMap Foundation, veröffentlich den Datenbestand unter der „Open Database Licens (ODbL); d.h., hier wird tatsächlich „,mit offenen Karten gespielt“.

Offene Geodaten sind aber nicht das einzige Thema des Kongresses. In der letzten Ausgabe (KN 02/19) haben wir das Programm vorgestellt. VR/AR sind innovative Technologien für viele Bereiche in Wirtschaft und Wissenschaft sind. Daher betrachten wir auch die Nutzung dieser Methoden in der Kartographie. Natürlich sind auch die klassischen Themen unseres Kongresses vertreten: Geoinformatik, Nutzen und Zugänge zu Geodaten, Geovisualisierung etc.

Kommen Sie in den Verbändepark der INTERGEO. Die DGfK wird mit einem eigenen Stand vertreten sein. Erfahren Sie die neuesten Entwicklungen in unserem Verein, informieren Sie sich über vergangene Veranstaltungen, zum Beispiel zur ICC in Tokyo, die im vergangenen Juli stattgefunden hat. Tauschen Sie sich mit anderen Mitgliedern unserer Gesellschaft aus, sprechen Sie mit dem Präsidenten, ihren Sektionsleiterinnen und Sektionsleitern. Reden Sie auch mit dem Schriftleiter der KN und teilen Sie mir Ihre Meinung zur ,neuen

Mark Vetter

mark.vetter@fhws.de

1 Wuerzburg, Deutschland

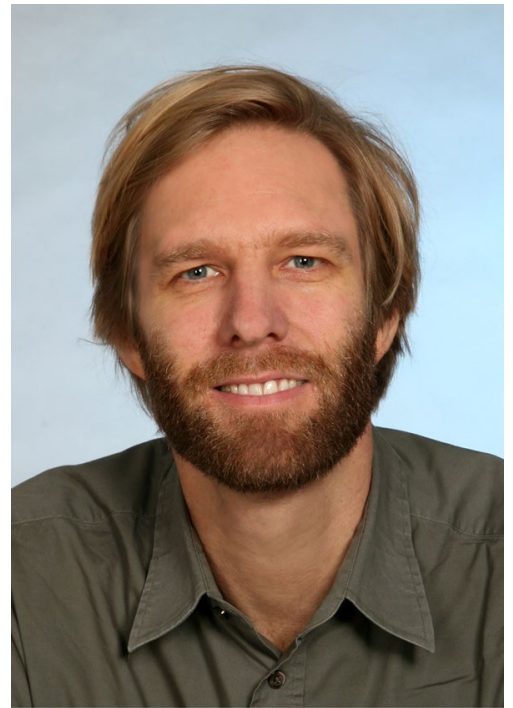

Schriftleiter: Mark Vetter

KN" mit, ähnlich wie dies die Autorinnen und Autoren in Leserbriefen in der vorliegenden Ausgabe getan haben!

Für die Mitglieder der DGfK ist der 16.9.2019 auch ein wichtiger Tag. Ein Teil des Vorstandes unserer Gesellschaft wird neu gewählt und Sie haben die Möglichkeit, in den Gremien unseres Vereins ihre Stimme zu erheben. Kommen Sie und bringen Sie sich ein. Geben Sie uns ein Feedback zu den neueren Entwicklungen in unserer Gesellschaft.

Viel Freude beim Lesen der aktuellen Ausgabe und auf ein Wiedersehen in Stuttgart.

Herzliche Grüße,

\section{Moruster}

Mark Vetter

Open Access This article is distributed under the terms of the Creative Commons Attribution 4.0 International License (http://creativeco mmons.org/licenses/by/4.0/), which permits unrestricted use, distribution, and reproduction in any medium, provided you give appropriate credit to the original author(s) and the source, provide a link to the Creative Commons license, and indicate if changes were made. 